\section{TOLERABILITY OF TIVOZANIB VS. SORAFENIB IN ELDERLY AND/OR IMMUNOTHERAPY-PRETREATED PATIENTS WITH METASTATIC RENAL CELL CANCER (MRCC) IN TIVO-3}

${ }^{1}$ Vijay Kasturi, ${ }^{2}$ Bernard Escudier, ${ }^{3}$ Brian Rini, ${ }^{4}$ Sumanta Pal ${ }^{*}$, ${ }^{5}$ David McDermott, ${ }^{6}$ Camillo Porta, ${ }^{7}$ Elena Verzoni. ' Aveo Oncology, Boston, MA, USA; ${ }^{2}$ Institute Gustave Roussy, Villejuif, France; ${ }^{3}$ Vanderbilt-Ingram Cancer Center, Nashville, TN, USA; ${ }^{4}$ City of Hope Comprehensive Cancer Center, Duarte, CA, USA; ${ }^{5}$ Beth Israel Deaconess Medical Center, Boston, MA, USA; ${ }^{6}$ Aldo Moro University of Bari, Bari, Italy; ${ }^{7}$ IRCCS Istituto Nazionale dei Tumori, Milan, Italy

Background The TIVO-3 trial demonstrated improved progression-free survival (PFS) with TIVO when compared to sorafenib (SOR; 5.6 mo. vs 3.9 mo., respectively; HR 0.73) and better tolerability with reduced need for dose interruptions ( $p$ $=0.0164)$, dose reductions $(\mathrm{p}=0.0147)$, and discontinuations $<\sup >1</$ sup $>$. As the majority of patients diagnosed with mRCC in the US are $>65$ years, with the largest recent increase in incidence among those $\geq 75$, and front-line treatment now standardly includes immunotherapy (IO), tolerability of new therapies for relapsed or refractory (R/R) mRCC must be acceptable in the elderly and/or IO pretreated population

Methods Data was analyzed to identify relationships between tolerability and advanced age or IO pretreatment. In addition to measures of drug exposure, any grade $\geq 3$ treatment related adverse events (TRAEs) and VEFGR TKI class effect grade $\geq 3$ TRAEs are reported by age $(<65,65-74, \geq 75)$ and prior IO (yes, no)

Results Of the 343 patients treated on study, 120 (35\%) were between age 65 and 75 and $34(10 \%)$ were over 75 . Patients received 1.5-2x more cycles of TIVO compared to SOR and fewer overall grade $\geq 3$ TRAEs in all age groups and irrespective of prior IO. Differences in VEGFR TKI class effect TRAEs seen in the total population were retained across most subgroups (table 1). Among patients 75 and over, there were almost half the rate of the dose reductions or discontinuations with TIVO compared to SOR. Prior IO was associated with less asthenia overall, more HTN with TIVO, and more rash but less diarrhea with SOR

Abstract 349 Table 1 Drug exposure, dose modifications, and TRAEs in TIVO-3 by age and prior 10

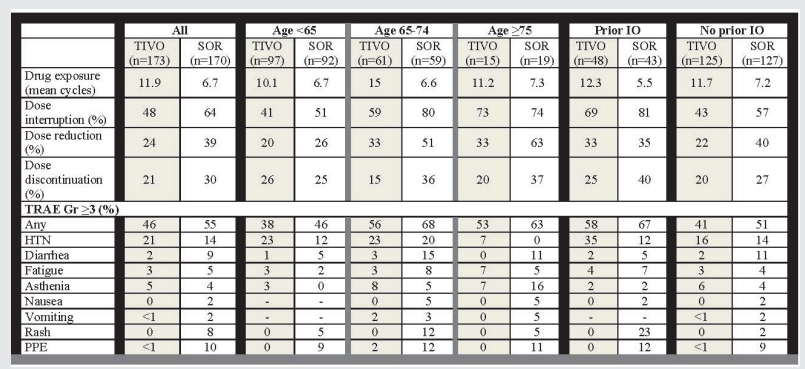

Conclusions Tolerability benefits with TIVO compared to SOR in $\mathrm{mRCC}$ are retained in elderly patients and those previously treated with IO. This finding, paired with consistently improved PFS in these subpopulations (age >65: HR 0.59, prior IO: HR 0.55), suggests TIVO is a safe and effective option in the context of the current $\mathrm{R} / \mathrm{R}$ mRCC treatment paradigm

Trial Registration ClinicalTrials.gov Identifier: NCT02627963

\section{REFERENCE}

1.. Rini $B$, Pal $S$, Escudier $B$, Atkins $M$, Hutson $T$, et al. Tivozanib versus sorafenib in patients with advanced renal cell carcinoma (TIVO-3): a phase 3, multicentre, randomised, controlled, open-label study. Lancet Oncol 2020;21:95-104

Ethics Approval This trial was approved by the institutional review board or ethics committee at every centre and complied with Good Clinical Practice guidelines, the Declaration of Helsinki, and local laws. All patients provided written informed consent before any trial procedure. The trial protocol including the relevant centres is provided in the appendix of the reference 1

http://dx.doi.org/10.1136/jitc-2021-SITC2021.349 